\title{
Proximal tibiofibular dislocation
}

\author{
J Horan, G Quin
}

Emerg Med J 2006;23:e33 (http://www.emjonline.com/cgi/content/full/23/5/e33). doi: 10.1136/emj.2005.032144

Proximal tibiofibular joint dislocation is an uncommon injury, which may be easily missed on plain radiography. If recognised, it can be treated in the emergency department, avoiding surgery and long term problems. The case is presented of a 22 year old male rugby player who was tackled from the left hand side while turning to the left. He heard a "pop" from his knee as he fell to the ground. Clinical examination revealed a prominence in the area of the fibular head. There was no evidence of peroneal nerve injury. Plain $x$ rays confirmed a clinical suspicion for anterior dislocation of the proximal tibiofibular joint. Proximal tibiofibular joint dislocation typically occurs when the knee is slightly flexed and the foot is rotated and plantar flexed. Antero lateral dislocation is the most common pattern. Diagnosis is largely clinical, but the findings may be subtle. Plain films may not show any abnormality and computed tomography is the investigation of choice if there is clinical suspicion for the injury. The dislocation should be reduced in the emergency department, but controversy exists whether early mobilisation or casting is the most appropriate course of action.

P roximal tibiofibular joint dislocation is an uncommon injury, which may be easily missed on plain $x$ rays. ${ }^{12}$ If recognised, it can be simply treated in the emergency department, avoiding surgery and long term problems. ${ }^{3}$

\section{CASE HISTORY}

A 22 year old male was brought to the emergency department (ED) by ambulance following an injury sustained on the rugby field. During the match he was tackled from the left hand side while turning to the left. He heard a "pop" coming from his knee while he fell to the ground, followed by immediate onset of pain in his left knee. He had no other injuries. On arrival in the ED, he had a vacuum splint in situ, supporting his left leg.

Examination of the left knee revealed tenderness to palpation. A prominence was visible in the area of the fibular head. There was full range of movement of the knee joint, but knee flexion accentuated the pain. The prominence was accentuated on knee flexion, and the head of the fibula became clearly outlined. He was able to bear weight satisfactorily and there was no foot drop or other evidence of a common peroneal nerve injury.

Plain films were obtained of the knee, tibia, and fibula (fig 1) plus ankle joint. These showed the fibular head to be displaced anterior and lateral to the tibiofibular joint. Comparison views were also taken, to confirm the diagnosis of anterolateral dislocation of the fibular head. There was no other injury noted. He was placed in a back slab and referred to the orthopaedic clinic after eight days, at which time the joint had reduced spontaneously.

\section{DISCUSSION}

The proximal tibiofibular joint is an inherently stable joint, due to good ligamentous support. ${ }^{4}$ This is especially true when the knee is in extension, as the joint is protected by the surrounding ligaments. Typically, dislocation occurs when the knee is held in flexion and the foot is rotated and plantar flexed. Isolated dislocations are seen in sports involving aggressive twisting of the knee. The injury has been reported in soccer, parachuting, ${ }^{4}$ snow-boarding, ${ }^{1}$ the long jump, ${ }^{2}$ horse riding, and in direct trauma.

Four injury patterns have been described. ${ }^{3}$ Subluxation occurs in preadolescent girls and is usually atraumatic. ${ }^{4}$ Anterolateral dislocation is the commonest injury pattern, accounting for $85 \%$ of dislocations. It is usually described after a fall with the knee flexed, the ankle inverted, and foot plantar flexed. Posteromedial dislocation occurs in $10 \%$. The mechanism is usually direct trauma, such as a blow from the bumper of a car or banging the knee against a gate post while horse riding. This is associated with a transient common peroneal nerve injury. Superior dislocation occurs in approximately $2 \%$ and is most often associated with high energy ankle injuries and also common peroneal injury.

Diagnosis is largely clinical, relying on suspicion for the injury. ${ }^{1}$ The findings on examination may be quite subtle, and are easily missed. ${ }^{2}$ Clinical features may include: lateral knee

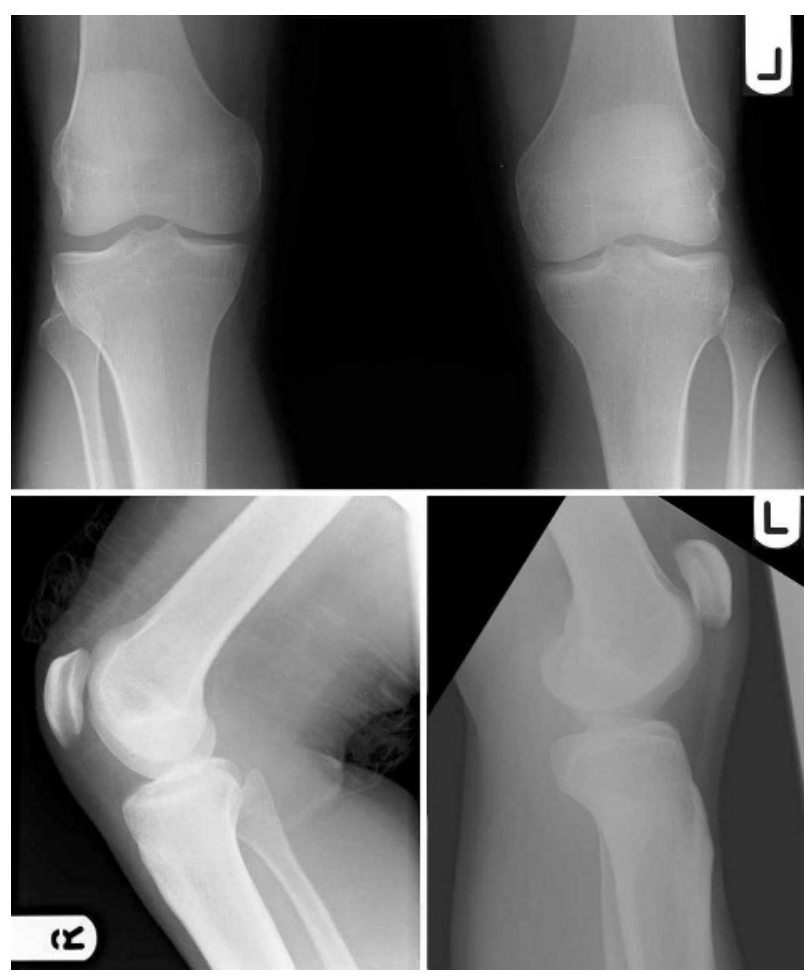

Figure $1 \quad X$ rays of left knee showing anterior dislocation of proximal tibiofibular joint with comparison views of a normal right knee. 
pain aggravated by pressure over the fibular head, limited knee extension, crepitus, visual deformity, and locking or popping. ${ }^{45}$ Ankle movement may exacerbate knee pain. ${ }^{4}$ There may be transient peroneal nerve palsy, especially with posterior or superior dislocations. ${ }^{4}$

Plain radiography has been shown to be inconsistent in aiding diagnosis, and studies suggest that computed tomography is the investigation of choice, if the injury is suspected. ${ }^{6}$ On plain films, careful evaluation of the congruity of the proximal tibiofibular joint, especially on the lateral view, is the key to diagnosis. ${ }^{7}$ In a normal knee, the fibular head overlaps the lateral margin of the lateral tibial condyle on the anteroposterior view, whereas on the lateral film the majority of the fibular head lies posterior to the posterior margin of the tibia.

Treatment of an anterolateral injury begins with closed reduction of the dislocation. ${ }^{3}$ The knee is flexed and the foot dorsiflexed and externally rotated. Pressure is applied over the fibular head while the injury mechanism is reversed, until a "pop" is heard. ${ }^{4}$ Controversy exists over whether casting is required or a Robert-Jones bandage and early mobilisation is sufficient. ${ }^{3}$ A case was reported of a young female who dislocated both proximal tibiofibular joints on different occasions, and had identical recovery from both treatment approaches. Posteromedial and superior dislocations, along with failed closed reduction of anterolateral injuries are repaired by open reduction and internal fixation. ${ }^{4}$
Authors'

J Horan, G Quin, Emergency Department, Mid-Western Regional Hospital, Dooradoyle, Limerick, Ireland

Competing interests: none declared

Written consent was obtained from the patient to have his clinical details published in the paper and online versions of the journal.

Correspondence to: Dr J Horan, Ballycar, Newmarket-on-Fergus,

County Clare, Ireland; jasonh@o2.ie

Accepted for publication 29 November 2005

\section{REFERENCES}

1 Ellis C. A case of isolated proximal tibiofibular joint dislocation while snowboarding. Emerg Med J 2003;20:563-4.

2 Laing AJ, Lenehan B, Ali A, et al. Isolated dislocation of the proximal tibiofibular joint in a long jumper. Br J Sports Med 2003;37:366-7.

3 Aladin A, Lam KS, Szypryt EP. The importance of early diagnosis in the management of proximal tibiofibular dislocation: a 9-and 5-year follow-up of a bilateral case. Knee 2002;9:233-6.

4 Wheeless' Textbook of Orthopaedics website: www.wheelessonline.com/ $001 / 67 \mathrm{u}, \mathrm{htm}$ (accessed 1 February 2005).

5 Sekiya JK, Kubn JE. Instability of the proximal tibiofibular joint. J Am Acad Orthop Surg 2003;11:120-8.

6 Keogh P, Masterson E, Murphy B, et al. The role of radiography and computed tomography in the diagnosis of acute dislocation of the proximal tibiofibular joint. Br J Radiol 1993;66:108-11.

7 Capps GW, Hayes CW. Easily missed injuries around the knee. Radiographics 1994;14:1191-210. 